\title{
Data collection protocols for wireless sensor networks
}

\author{
Mohamed Labib Borham \\ Helwan University, \\ Research Support Centre in Computing and Informatics (RSCCI), \\ Faculty of Computers and Artificial Intelligence, Computer Science Department \\ Cairo, Egypt \\ mlabib@msa.eun.eg
}

\section{Ghada Khoriba}

Helwan University,

Faculty of Computers and Artificial Intelligence, Computer Science Department

Cairo, Egypt

ghada_khoriba@fci.helwan.edu.eg

\section{Mostafa-Sami Mostafa}

Helwan University,

Faculty of Computers and Artificial Intelligence, Computer Science Department

Cairo, Egypt

mostafa.sami@fci.helwan.edu.eg

\begin{abstract}
Data collection in wireless sensor networks (WSNs) has a significant impact on the network's performance and lifetime. Recently, several data collection techniques that use mobile elements (MEs) have been recommended, especially techniques that focus on maximising data delivery. However, energy consumption and the time required for data collection are significant for many WSN applications, particularly real-time systems. In this paper, a review of data collection techniques is presented, providing a comparison between the maximum amount shortest path (MASP) and zone-based energy-aware (ZEAL) data collection protocols implemented in the NS-3 simulator. Finally, the study provides a suitable data collection strategy that satisfies the requirements of WSN applications in terms of data delivery, energy consumption, and the time required for data collection.
\end{abstract}

Keywords: wireless sensor networks, data collection, routing protocols, mobility

\section{INTRODUCTION}

Wireless sensor networks (WSNs) were introduced in 1994. A WSN is an ad hoc network that collects data of the deployment area. WSNs are used in various applications, especially in environment monitoring applications [1].

WSNs consist of independent devices called sensors or nodes, of which there may be thousands in largescale networks. Sensors have many components for processing, sensing, power supply, and other functions. The nodes in WSNs have limited resources for processing, energy, memory, and bandwidth. Therefore, it is important to use these resources wisely.

Sensors collect and process the environment's data and send it to their neighbouring nodes (intermediate nodes) to reach the destination (base station). According to sensor type, two types of networks can be distinguished: homogenous and heterogeneous WSNs.
Homogenous WSNs consist of identical nodes in terms of their capabilities. Heterogeneous WSNs, in contrast, have more than one type of sensor [2]. Fig. 1. shows the structure of a WSN.

Data collection operations mainly consume network energy, so energy management is vital for prolonging a network's lifetime. Therefore, using an effective data collection strategy should be considered to enhance a WSN's efficiency.

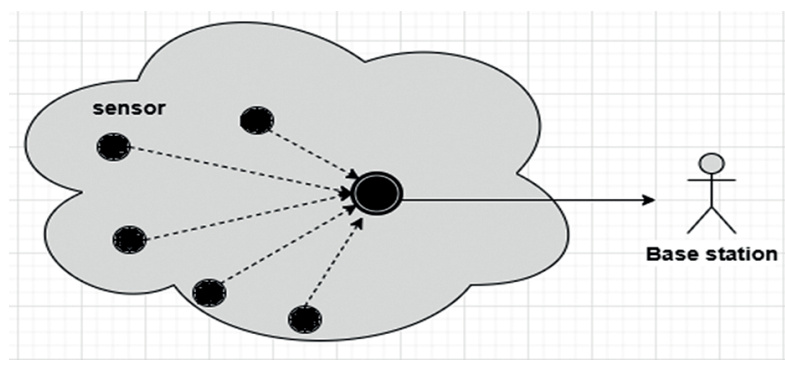

Fig. 1. Wireless sensor network 
Many WSN designs distribute the energy consumption between the deployed sensors according to their locations and the location of the sink node (or its trajectory if it is a mobile data collector). In addition, they specify the periods in which the sensors are active or at standby to save energy.

Although the main goal of the data collection strategy is to collect the maximum amount of data, the time required for the data collection has a significant effect on energy consumption. This issue will be discussed in more detail in the following sections.

The structure of the rest of the paper is as follows. Section 2 presents the routing protocols in WSNs and provides their challenges. Section 3 presents the concepts of clustering in WSNs. Mobility and WSNs are introduced in Section 4. The data collection process in WSNs and the related mobility issues are discussed in Section 5. A review of the data collection studies is covered in Section 6. A detailed comparison between the maximum amount shortest path (MASP) and zonebased energy-aware (ZEAL) data collection protocols is given in Section 7. Finally, conclusions and future work are mentioned in Section 8.

\section{ROUTING PROTOCOLS IN WSNS}

Routing protocols are one of the most significant research areas in WSNs. Routing in WSNs faces some challenges that are a trade-off between responsiveness and efficiency. This balance must deal with limited communication capabilities and sensor suspension versus the overhead required to adapt to this situation. In a WSN, the total overheads are calculated primarily on the basis of bandwidth usage, energy consumption, and processing requirements for sensor nodes. A strategy for balancing these parameters is the basis for testing the routing efficiency.

Routing protocols in ad hoc networks can be divided into categories depending on the way the data are captured and the manner in which paths are estimated on the basis of the captured data. Routing protocols can be categorised as follows [3]:

\subsection{PROACTIVE ROUTING}

The proactive routing protocols require that the information is spread according to accurate routing tables. The sensing nodes set their routing tables before the sink nodes request the data. The routing tables are updated according to the network's topology structure.

The network's structure may be flat or hierarchical. In the flat type, the nodes send their data to reach the sink node through intermediate nodes using multi-hops. In contrast, in the hierarchical type, the network is organised in adjacent groups called clusters. Each node in a cluster sends its data to the cluster head to forward it to the sink node. The hierarchical type is often used in large-scale WSNs for saving energy. Clustering will be presented in detail in Section 3.

\subsection{REACTIVE ROUTING}

Reactive routing creates dynamic paths from the sensing nodes to the sink node according to the application's requirements. The routing path selection does not depend on the global information of the network or the routing tables. This type of routing usually involves flooding a discovery query. The use of flooding raises the number of messages through the network. As a result, the number of connections increases, which increases energy consumption. Therefore, it is vital to use a method for controlling the flooding process.

\subsection{HYBRID ROUTING}

In WSNs, many applications use a mix of reactive and proactive routing, which is called a hybrid routing protocol. Hybrid routing protocols rely on the network's architecture for stability and scalability in large-scale networks.

\section{CLUSTERING AND WSNS}

The communication process in a WSN is an essential factor in energy consumption. Numerous researchers are trying to find an efficient communication method to reduce energy consumption. They try to decrease the number of communication messages to decrease the total communication overload. One of the wellknown models is clustering.

The clustering components are the following [4]:

- Sensor node: the essential component of a WSN. It has the capability of sensing and processing the data.

- $\quad$ Cluster head $(\mathrm{CH})$ : the coordinator for a selected group (cluster). The $\mathrm{CH}$ is responsible for many activities in the group, such as data collection and data transfer to the base station.

- Base station: the central node for data collection in a WSN. It is the ultimate source of data for the end-user of the network.

- Cluster: the network's structural unit that is used to facilitate communication in WSNs.

In the clustering model, the sensing nodes send their data to the cluster head in their region. The cluster heads, in turn, forward their data to the base station. Using this strategy achieves a reduction in energy consumption and the number of transmitted messages. Fig. 2. shows the components of the cluster model.

Clustering has many advantages: it helps transfer the data to the base station, decreases the number of intermediate nodes involved in the transmission, saves energy, supports network scalability, and reduces data transmission. However, the main problem occurs when one of the cluster heads is down because of energy depletion [4]. 


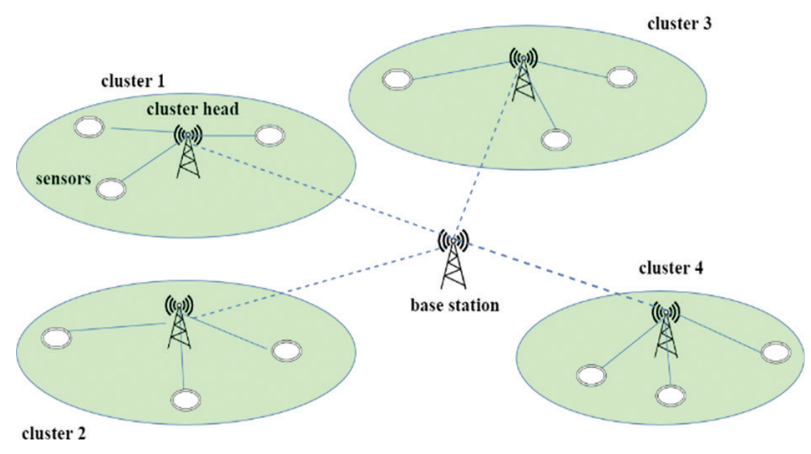

Fig. 2. Clustered sensor network

\section{WSNS WITH MOBILITY}

Many studies proposed mobility in the architecture of WSNs: mobility in sensing nodes or sink nodes or both. Using mobility can reduce the number of transferred messages and energy consumption.

Mobile wireless sensor networks (MWSNs) are different from the fixed WSNs. Incorporating navigation into network nodes can change many network features. The following features can be changed as a result of navigation $[5,6]$ :

- Localisation: In fixed sensor networks, the sensors are static as soon as they are deployed, so it is possible to obtain information about the location of the adjacent sensors. In MWSNs, the sensors move within the grid, so energy and transmission techniques are important for getting the location of the adjacent sensors.

- Network topology: Because of the continuous motion of the sensors, the routing tables are updated in each period. Note that most common WSN routing algorithms do not consider the dynamic changes in the routing tables used in MWSNs.

- Energy consumption: An MWSN requires dynamic motion between sensors, which consumes a large amount of energy. In the applications of WSNs, the energy consumption is taken care of by providing the nodes with a large source of power or recharging capability.

- Network sink: Most WSN types use a static sink node. However, in some cases, the sink can be mobile, as in an MWSN. This mobile sink node visits the sensor nodes to gather their data. Therefore, data collection techniques are different in an MWSN.

- Complexity: The algorithms used in an MWSN are more complex than those in a fixed WSN. The mobile sensor deployment mechanism is considered to be one of the biggest design challenges for the algorithms in MWSNs. In addition, a complicated node design is used in MWSNs.

The mobility approach provides many advantages in terms of connectivity, cost, reliability, and energy effi- ciency. However, using navigation in WSNs comes with many challenges that do not exist in fixed WSNs, such as connection discovery, perceived power management for mobility, reliable data transfer, and navigation control [6].

An MWSN contains two main components:

- Regular nodes, which acquire the environment data.

- Sink nodes, which receive the data from the other nodes.

The previous WSN-ME (mobile element) architecture is called homogeneous or flat. Otherwise, in some WSN-ME architectures, support nodes are used as an intermediate data collector or gateway. This WSN-ME architecture is called heterogeneous or tiered.

\section{DATA COLLECTION IN MWSNS}

Data collection is one of the most important research fields in WSNs. The sensors' energy is consumed mainly for data transmission through the network. Radio frequency (RF) shared broadcasting is responsible for up to $80 \%$ of the energy consumption, so many researchers are trying to reduce the number of transmitted messages.

The goal of the data collection protocol is to collect the maximum amount of data and reduce the data loss by considering energy consumption. Through effective data collection techniques, the performance and effectiveness of the network can be improved, thereby also prolonging the network's lifetime.

Traditional data collection protocols depend on collecting data directly from the sensing nodes or using intermediate nodes to forward the network's data to the sink node. The intermediate nodes utilise more energy than the other nodes and deplete faster. The traditional method is effective in data collection but has an impact on prolonging the network's lifetime, especially in large-scale networks. Additionally, these nodes are distributed randomly in a dynamic environment, so it is hard to use routing tables or to gather geographic information for each node.

The traditional protocols transfer huge messages, thereby consuming a lot of energy, so mobile data collectors (MDCs) have been introduced to solve these problems. The mobile collector moves on a pre-defined or random path to get the sensed data from the nodes within its communication range.

Mobility may be incorporated into the network's components. For example, the nodes may be moving, and the sink nodes may be static. This architecture is called a WSN-ME architecture with relocatable nodes. Converse$l y$, the nodes may be static, and the sinks may be mobile. This architecture is called a WSN-ME architecture with MDCs, which use a mobile sink or mobile relay. The network can be regarded as a WSN-ME architecture when at least one of the components listed above is mobile [6]. 
Data collection in a WSN-ME architecture is different from the data collection operations in traditional WSNs (static networks). Several main phases are needed to collect the data in WSN-ME architectures. Below, these phases are described together with their requirements and challenges [6].

- Discovery protocols: These protocols are used to detect contacts as soon as the connection becomes available. Many different approaches exist. In mobility-independent protocols, the MEs can be recognised by their navigation style (scheduled rendezvous, on-demand, and asynchronous). With prior knowledge about the mobility of nodes, the sensors are in an inactive mode and are activated when the ME device is expected to contact a node. Asynchronous detection protocols are used to verify that the ME is within the range of the network nodes. In this case, messages are constantly emitted from the ME while the fixed sensors are set ready. When the ME is detected, the sensors start storing the communication time to prepare their routing table.

- Data transfer protocols: These protocols are designed to maximise the number of successfully transmitted messages from each node while minimising energy consumption. The MDC speed and the distance between the MDC and the sensors affect the data collection ratio.

- $\quad$ Routing to mobile elements: When the network density is sufficient, multiple paths may be allowed. Routing techniques with uncontrollable MEs can be classified into two classes. In horizontal (flat) routing, all nodes operate with identical mechanisms and roles. In proxy-based routing, several agents or gates are chosen from the sensor nodes, and these agents are considered the means of communication between static sensors and MEs.

- Motion control: The node's movements are either under control or uncontrollable. With uncontrollable navigation, the sensors are compatible with the path of the ME across the network. When navigating is controllable, the ME's movements can be designed to achieve specific objectives and to improve the performance standards. Mobility can be improved by selecting an efficient trajectory for the ME's movements and speed.

\section{DATA COLLECTION METHODOLOGIES AND TECHNIQUES: AN OVERVIEW}

In this section, a review of data collection methodologies and techniques in MWSN are presented with their structures and concepts. Additionally, some open research areas are mentioned for future work.

Nguyen et al. [7] presented compressive sensing for data collection in WSNs using mobile sensors distributed randomly in the network. These mobile sensors move randomly in any direction with different velocities but within their neighbours' transmission range to exchange their sensed data. The sensors execute rounds of movements to collect data about the current position. Finally, they are able to reconstruct the data about the sensing area. However, using more than one mobile sensor requires additional techniques to handle the coordination between the nodes.

The clustering mechanism is successful in maintaining energy resources for network activities. Many unbalanced aggregation algorithms have been proposed to overcome the issue of hotspots (using intermediate nodes in a traditional WSN). However, the energy depletion problem remains because the activities of the head of the cluster are closely related to its position in the network. Clustering in an MWSN involves an additional challenge: how to control the motion of the ME to access the cluster heads when the model does not depend on routing tables.

Gattani et al. [8] proposed a data collection algorithm that depends on compressive sensing using scorebased load balancing. The sensed area is divided into clusters. Each cluster contains a head selected by the other nodes. The cluster head is selected from the two best nodes according to their remaining energy and the distance between them and the base station. The sensed data is compressed by compressive sensing in the cluster head and forwarded to the final destination. The network data is reconstructed from several measurements in the final destination. Through this model, the overall amount of transmitted data and energy consumption are reduced.

Du et al. [9] tried to overcome the memory limitation (or buffer-overflow) problem. The authors proposed a mobile-sink data collection approach that depends on heterogeneous sensors in which each sensor has its sampling rate, buffer size, and buffer-overflow time. The proposed algorithm divides the sensed area into groups (clusters). Each group contains a cluster head with buffer-overflow time. The buffer-overflow time is increased by adding a temporary cache to the cluster head as a deputy cluster head. Finally, they proposed an algorithm to determine a moving path to the mobile sink to reach the cluster head on time to prevent buffer-overflow problems.

Luo et al. [10] presented a two-tier data dissemination (TTDD) protocol that depends on building a grid-based structure. The grid structure consists of cells (squares) and crossing points (the intersections of the grid lines). In forwarding the data, the source sensor is considered a transit point that sends an announcement message to its four adjacent junctions, thereby reducing the spread of the network data by exploiting the location of the crossing points. For example, only the nearest node to the intersection will process the message. The repetitive publication spreads to the sensors and acts as a proxy to identify routing paths. The MDC begins the data collection process by sending a query message to its region. 
The deployment points publish the query within the network and retrieve the network data from the nodes. In a TTDD protocol, it is assumed that the nodes' positions are known, and the protocol takes advantage of the geographical location of the nodes.

Kumar et al. [11] presented a scheme for data collection in WSNs with an integer linear formulation. The sensed area is divided into grids that contain many nodes with a random distribution. Each grid has a cache point to receive the sensed data from the sensors in the same grid. After that, an ME collects the data from the cache points. The actions in the scheme are split into two parts. First, find the cache points. Secondly, find the shortest path between the cache points, and use it as the ME's path.

Gao et al. [12] presented the maximum amount shortest path (MASP) protocol. The MASP protocol is a data collection strategy that uses a mobile sink moving along a constrained path. The sensors in the communication range of the sink are chosen as gateways to gather the data from the other nodes using multi-hop routing. Integer linear programming with a genetic algorithm decreases the transmission steps from the sensing nodes to their destination (a sub-sink node). The authors mentioned the bottleneck problem, which occurs when a set of proxies has many messages with a short contact time with the ME (the problem of hot spots). This problem is handled by allocating proxy nodes according to the length of the contact time to increase the throughput with minimum energy consumption. They use a twophase data collection protocol, called ME discovery, and data gathering to implement this scheme. The MASP protocol operates with and supports the use of many MEs. By using the OMNET++ simulator, the authors show that this scheme performs better than the shortest path tree (SPT) method according to the amount of data collected and the energy consumption. The authors use three cycles in the discovery phase to identify the network topology and distribute the topology information to assign the members' nodes to their corresponding sub-sinks. Consequently, the data collection consumes overall a large amount of energy and time. Another issue is that the protocol depends on a synchronised transmission between the mobile sink and sub-sink nodes, thus requiring a perfectly synchronised clock between the nodes, which is not available in many systems.

Gallegos et al. [13] presented an implementation of the MASP routing protocol using a network simulator (NS-3). The authors show that the protocol uses three rounds (a mobile sink going back and forth to the beginning of its trajectory is called a round) to complete the data collection process. Their results show that the MASP routing protocol performs better in terms of energy consumption than the ad hoc on-demand distance vector (AODV) protocol.

Gallegos et al. [14] proposed an energy-aware data collection protocol (ZEAL) based on the MASP protocol. The protocol is based on dividing the environment into independent zones according to the movement path of the mobile sink. The mobile sink moves through a pre-defined trajectory to set routing zones and select sub-sink nodes. The mobile sink collects data from the sub-sinks that receive data from the sensors. After that, a communication time-slot assignment algorithm is used to assign the sub-sink nodes to the mobile sink to overcome the hot-spot issue. The mobile sink calculates the number of member nodes assigned to each sub-sink node according to their contact time. When the sub-sink nodes receive the number of nodes assigned to them, they send it to each member node to calculate the priority to select one sub-sink node and send their data to the targeted sub-sink. The mobile sink goes inside the network to collect the sensed data from the sub-sinks. In addition, the member nodes use duty cycling to reduce energy consumption. The authors presented an implementation of the protocol using the NS-3 simulator. The results show that the performance of the ZEAL protocol is better than that of the MASP protocol in terms of both energy consumption and packet delivery rate.

\section{MASP AND ZEAL PROTOCOLS}

In this section, the MASP [12] and ZEAL [14] protocols are compared in detail.

\subsection{MAIN FEATURES}

The MASP and ZEAL protocols use the following three types of nodes (Fig. 3.):

- $\quad$ Sink: a node that collects data from the sub-sink nodes.

- Sub-sink: a node that is within the communication range of a sink node and that is in direct connection with it.

- Member: a node that is not in direct connection with a sink and that uses sub-sink nodes as intermediate nodes.

The MASP and ZEAL protocols use random node deployment, hybrid routing protocols, and multi-hop communication modes. In addition, they can be applied in large-scale WSNs. Table 1 shows the different features of the ZEAL and MASP protocols in detail.

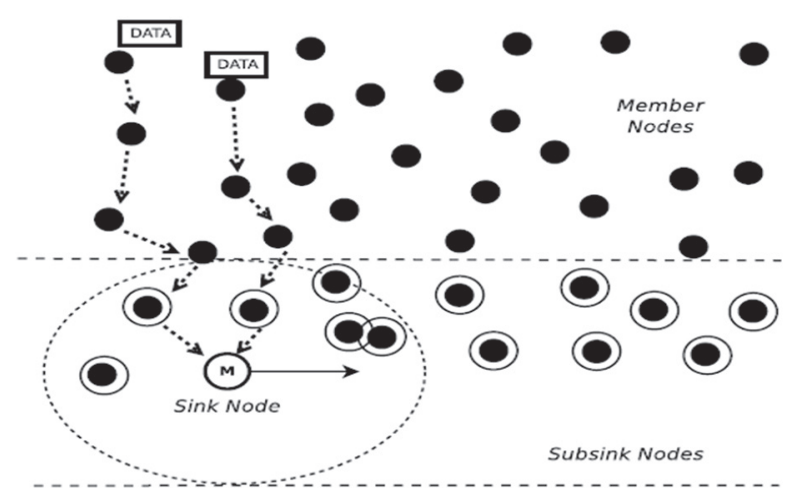

Fig. 3. Node types [14] 
Table 1. Different features of the MASP and ZEAL protocols.

\begin{tabular}{|c|c|c|}
\hline Feature & MASP & ZEAL \\
\hline Routing type & Proactive & Hybrid \\
\hline Number of cycles & 3 & 2 \\
\hline Number and names of rounds & $\begin{array}{c}2 \\
\text { Discover, data collection }\end{array}$ & $\begin{array}{c}2 \\
\text { Setup, data collection }\end{array}$ \\
\hline First phase & Assigning members to sub-sinks & $\begin{array}{l}\text { Creating independent zones, populating } \\
\text { routing, and assigning a sub-sink to each zone. }\end{array}$ \\
\hline Second phase & $\begin{array}{l}\text { Member nodes start collecting data from } \\
\text { the monitoring area to send the data to the } \\
\text { assigned sub-sink, and the sub-sink sends its } \\
\text { data to the mobile sink }\end{array}$ & $\begin{array}{l}\text { Member nodes start collecting data from their } \\
\text { zone to send the data to the assigned sub-sink, } \\
\text { and the sub-sink sends its data to the mobile } \\
\text { sink }\end{array}$ \\
\hline Method to assign sub-sinks & According to the length of the contact time & According to the number of zones \\
\hline $\begin{array}{c}\text { Methods to solve overlap in communication } \\
\text { contact time }\end{array}$ & $\begin{array}{c}\text { Minimum, shared, or optimally shared } \\
\text { overlapping time }\end{array}$ & Minimum, shared, or selective time assignment \\
\hline Method to assign member nodes to sub-sinks & $\begin{array}{l}\text { A centralised heuristic solution based on a } \\
\text { genetic algorithm }\end{array}$ & $\begin{array}{l}\text { Member requirements in terms of number of } \\
\text { hops }\end{array}$ \\
\hline Zone partitioning & No & Yes, automatic \\
\hline Data collection mechanism & Synchronised & Poll \\
\hline Energy saving & No & Yes \\
\hline
\end{tabular}

The main discussion point is how the time required for the data collection operations affects the overall data collection performance in terms of data delivery and energy consumption.

In $[12,14]$, the authors use the terms cycle and round with the same meaning. A cycle is defined as the tour of a mobile sink from the start point of the path to the end and back to the start point again.

According to this definition of a cycle, the total data collection time $\left(T_{c o l}\right)$ is

$$
T_{\text {col }}=N \times C_{\text {time }}
$$

where $\mathrm{N}$ is the number of cycles and Ctime is the time for one cycle.

Gao et al. [12] presented the MASP protocol and proposed a data collection process that is completed within three cycles for ME discovery only, another cycle for the data collection process.

Gallegos et al. [13] presented an implementation of the MASP protocol using the NS-3 simulator and proposed that the data collection process can be achieved within three cycles.

Gallegos et al. [14] presented the ZEAL protocol. The data collection process can be achieved within two cycles (one cycle for setting up the network and another cycle for the data collection process). However, the authors use three cycles (one cycle for setting up the network and two cycles for data collection) in the simulation's implementation.

In the next section, the MASP and ZEAL protocols are implemented with a well-known simulator (NS-3) to show the effect of the time-saving method on data delivery and energy consumption.

\subsection{IMPLEMENTATIONS AND EVALUATION OF THE RESULTS}

The MASP and ZEAL protocols are implemented in the NS-3 simulator, using randomly distributed nodes. The network area is assumed to be a rectangle ( $400 \times 200$ $\mathrm{m})$. The mobile sink moves on a constrained path at the bottom of the rectangle. The initial energy of the nodes is $3000 \mathrm{~J}$ with a $52-\mathrm{m}$ communication range. The speed of the mobile sink is $5 \mathrm{~m} / \mathrm{s}$. The data transmission rate from a sub-sink to a mobile sink is $20000 \mathrm{bps}$; the data transmission rate from a member node to a sub-sink is 8232 bps.

In this section, the data collection performance of the MASP protocol (with three cycles) and that of the ZEAL protocol (with two and three cycles) is compared in terms of

- Data delivery (Data Del): the percentage of the delivered data (the sink node) relative to the total number of nodes.

- $\quad$ Average of the remaining energy (Avg Re Eng): the percentage of the average remaining energy relative to the initial energy.

According to Eq. (1), the number of cycles affects the total data collection time. In the data collection process, the ZEAL protocol saves one movement cycle of the mobile sink, so the ZEAL protocol saves 33\% of the total time needed for the data collection. This saving is highly relevant in many WSN applications, especially in emergency applications.

Figs. 4 and 5 show that the ZEAL protocol with two cycles is better in saving energy than the MASP and ZEAL protocols with three cycles. This saving occurs by reducing the time needed for data collection and the number of transmitted messages. Therefore, if the objective of a WSN application is saving energy, then it is better to use the ZEAL protocol with two cycles only. 
In contrast, the MASP and ZEAL protocols with three cycles are better in data delivery than the ZEAL protocol with two cycles.

Figs. 4 and 5 show the trade-off between data delivery and the average reaming energy, indicating that maximising the data delivery implies more energy consumption. In contrast, increasing the time of data collection improves the data delivery.

Fig. 6 shows that the MASP protocol is better than the ZEAL protocol in data delivery with an increasing number of nodes. The results show that the MASP protocol is more suitable for large-scale networks.

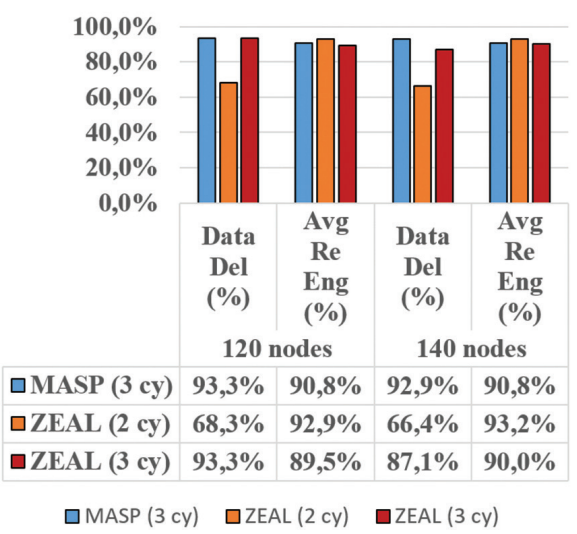

Fig. 4. MASP and ZEAL with 120 or 140 nodes

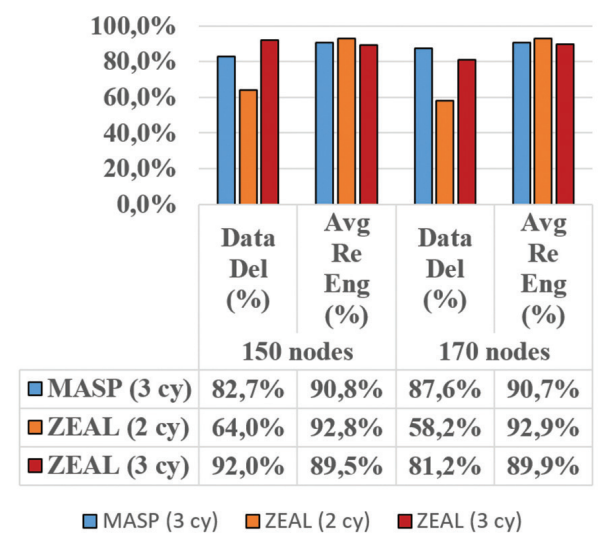

Fig. 5. MASP and ZEAL with 150 or 170 nodes

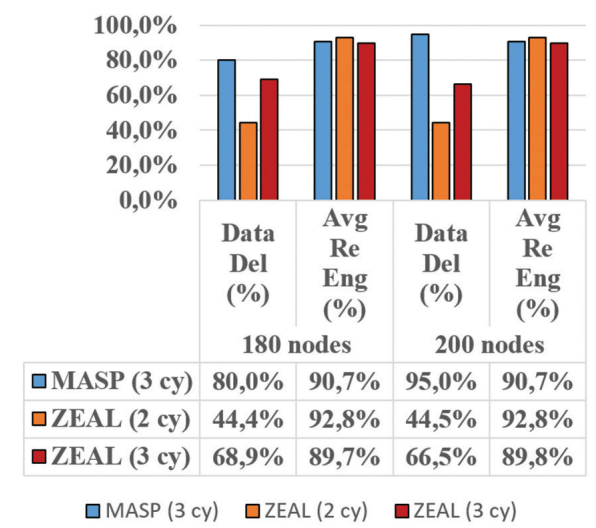

Fig. 6. MASP and ZEAL with 180 or 200 nodes

\section{CONCLUSION AND FUTURE WORK}

Using mobility in data collection improves the energy consumption and affects the network's lifetime. However, mobility causes new challenges, such as routing the mobile sink, contact discovery, data transfer, and motion control.

Recently, zone-based protocols have proved to be efficient in the data collection process while minimising energy consumption.

This study presented a detailed comparison between the MASP and ZEAL protocols, which will be helpful for researchers in selecting the most suitable protocol for a WSN application. The results indicated that the ZEAL protocol with two cycles saved $33 \%$ of the total time needed for the data collection in the MASP protocol, therefore, it is suitable for emergency applications.

However, if time is not critical, the MASP protocol with three cycles can be used to maximise the data delivery. In addition, the results showed that the MASP protocol is more suitable for large-scale networks.

Future work will be related to developing zone-based protocols using artificial intelligence features to improve the contact discovery and data transfer methods.

\section{REFERENCES}

[1] I. F. Akyildiz, W. Su, Y. Sankarasubramaniam, E. Cayirci, "A Survey on Sensor Networks", IEEE Communications Magazine, Vol. 40, No. 8, 2002, pp. 102114.

[2] K. Akkaya, M. Younis, "A Survey on Routing Protocols for Wireless Sensor Networks", Ad Hoc Networks, Vol. 3, No. 3, 2005, pp. 325-349.

[3] R. R. Ema, A. Anik, N. Nahar, M. Rahman, K. Eti, T. Islam, "Simulation Based Performance Analysis of Proactive, Reactive and Hybrid Routing Protocols in Wireless Sensor Network", Proceedings of the $11^{\text {th }}$ International Conference on Computing, Communication and Networking Technologies, Kharagpur, India, 1-3 July 2020, pp. 1-6.

[4] J. Patole, J. Abraham, "Design of MAP-REDUCE and K-MEANS Based Network Clustering Protocol for Sensor Networks", Proceedings of the $3^{\text {rd }}$ International Conference on Computing, Communication and Networking Technologies, Coimbatore, India, 26-28 July 2012, pp. 1-5.

[5] A. Boukerche, "Algorithms and Protocols for Wireless Sensor Networks", $1^{\text {st }}$ Edition, Wiley-IEEE Press, 2008. 
[6] M. Francesco, S. Das, G. Anastasi, "Data Collection in Wireless Sensor Networks with Mobile Elements: A Survey", ACM Transactions on Sensor Networks, Vol. 8, No.1, 2011, pp. 1-3.

[7] M. Nguyen, K. Teague, S. Bui, "Compressive Wireless Mobile Sensing for Data Collection in Sensor Networks", Proceedings of the International Conference on Advanced Technologies for Communications, Hanoi, Vietnam, 12-14 October 2016, pp. 437-441.

[8] V. Gattani, S. Jafri, "Data Collection Using Score Based Load Balancing Algorithm in Wireless Sensor Networks", Proceedings of the International Conference on Computing Technologies and Intelligent Data Engineering, Kovilpatti, India, 7-9 January 2016, pp. 1-3.

[9] J. Du, H. Wang, Y. Wu, F. Jiang, H. Huang, "A Data Collection Approach Based on Mobile Sinks for Heterogeneous Sensor Networks", Proceedings of the 8th International Conference on Wireless Communications \& Signal Processing, Yangzhou, China, 13-15 October 2016, pp. 1-6.

[10] H. Luo, F. Ye, J. Cheng, S. Lu, L. Zhang, "TTDD: TwoTier Data Dissemination in Large-Scale Wireless Sensor Networks", Wireless Networks, Vol 11, No. 1, 2005, pp. 161-175.
[11] S. Kumar, R. Chaudhary, A. Deepak, D. Dash, "An Integer Linear Formulation Scheme for Data Collection in Wireless Sensor Network Using Mobile Element (ME)", Proceedings of the $13^{\text {th }}$ International Conference on Wireless and Optical Communications Networks, Hyderabad, India, 21-23 July 2016, pp. 1-6.

[12] S. Gao, H. Zhang, S. Das, "Efficient Data Collection in Wireless Sensor Networks with Path-Constrained Mobile Sinks", Proceedings of the IEEE International Symposium on a World of Wireless, Mobile and Multimedia Networks \& Workshops, Kos, Greece, 15-19 June 2009, pp. 1-9.

[13] A. Gallegos, T. Noguchi, T. Izumi, Y. Nakatani, "Simulation Study of Maximum Amount Shortest Path Routing in Wireless Sensor Networks Using Ns-3", Proceedings of the 8th International Conference on Ubiquitous and Future Networks, Vienna, Austria, 5-8 July 2016, pp. 198-204.

[14] A. Gallegos, T. Noguchi, T. Izumi, Y. Nakatani, "Zone-Based Energy Aware Data Collection Protocol for WSNs", IEICE Transactions on Communications, Vol. E101.B, No. 3, 2018, pp. 750-762. 